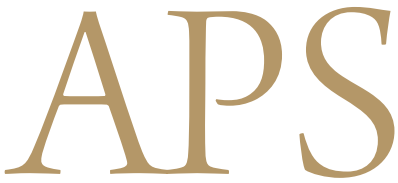

Archives of Plastic Surgery

\title{
Validation of the optimal scaffold pore size of nasal implants using the 3-dimensional culture technique
}

\author{
Jeoung Hyun Nam, So Yun Lee, Galina Khan, Eun Soo Park \\ Department of Plastic and Reconstructive Surgery, Soonchunhyang University Bucheon Hospital, Bucheon, Korea
}

Background To produce patient-specific nasal implants, it is necessary to harvest and grow autologous cartilage. It is crucial to the proliferation and growth of these cells for scaffolds similar to the extracellular matrix to be prepared. The pore size of the scaffold is critical to cell growth and interaction. Thus, the goal of this study was to determine the optimal pore size for the growth of chondrocytes and fibroblasts.

Methods Porous disc-shaped scaffolds with 100-, 200-, 300-, and 400- $\mu \mathrm{m}$ pores were produced using polycaprolactone (PCL). Chondrocytes and fibroblasts were cultured after seeding the scaffolds with these cells, and morphologic evaluation was performed on days 2,14 , 28 , and 56 after cell seeding. On each of those days, the number of viable cells was evaluated quantitatively using an MTT assay.

Results The number of cells had moderately increased by day 28. This increase was noteworthy for the 300- and 400- $\mu \mathrm{m}$ pore sizes for fibroblasts; otherwise, no remarkable difference was observed at any size except the 100- $\mu$ m pore size for chondrocytes. By day 56 , the number of cells was observed to increase with pore size, and the number of chondrocytes had markedly increased at the $400-\mu \mathrm{m}$ pore size. The findings of the morphologic evaluation were consistent with those of the quantitative evaluation.

Conclusions Experiments using disc-type PCL scaffolds showed (via both morphologic and quantitative analysis) that chondrocytes and fibroblasts proliferated most extensively at the $400-\mu \mathrm{m}$ pore size in 56 days of culture.

Keywords Porosity / Rhinoplasty / Artificial implant / Chondrocyte / Fibroblast
Correspondence: Eun Soo Park Department of Plastic and Reconstructive Surgery, Soonchunhyang University Bucheon Hospital, 170 Jomaru-ro, Wonmi-gu, Bucheon 14584, Korea Tel: +82-32-621-5319 Fax: +82-32-621-5016 E-mail: peunsoo@schmc.ac.kr

This work was supported by the Soonchunhyang University Research Fund.

This article is a part of Jeoung Hyun Nam's M.D. thesis in 2019 at Soonchunhyang University Graduate School.

Cell morphology was visualized using a Hitachi SU8220 field-emission scanning electron microscope at the Western Seoul Center of the Korea Basic Science Institute.

Received: February 14, $2020 \bullet$ Revised: May 19, $2020 \bullet$ Accepted: May 19, 2020

pISSN: 2234-6163 • elSSN: 2234-6171 • https://doi.org/10.5999/aps.2020.00213• Arch Plast Surg 2020;47:310-316

\section{INTRODUCTION}

In Asians, the nose is generally marked by features including a low wide dorsum, a retracted columella, poor projection of the nasal tip, and weak cartilaginous support [1]. Thus, most rhinoplasty procedures for Asian patients involve augmentation. The implants used in dorsal augmentation can be broadly divided into autologous and alloplastic implants. Among the latter, ex- panded polytetrafluoroethylene and silicone are commonly utilized as primary options, especially for Asian patients [2]. However, silicone is associated with complications, such as foreign body reactions, infection, and protrusions. Thus, surgery using autologous tissue, which has minimal side effects, is recommended; however, this technique also has disadvantages, including the finite amount of source tissue available, the need for additional surgery to obtain autologous cartilage, the long oper- 
ation time, and donor site morbidity [3].

To overcome these drawbacks, studies have reported the production of autologous tissue using bioengineering technology. A study was conducted on the production of poly-L-lactic acid scaffolds using a mold method, along with the production of alar cartilage via the implantation of chondrocytes [4]. The use of patient-specific nasal implants has also been reported in a study in which a cartilage-derived hydrogel was injected into a polycaprolactone (PCL) scaffold [5].

One of a number of scaffold materials, $\mathrm{PCL}$ is a synthetic polymer that is widely used in tissue engineering and that has been demonstrated to be safe in vivo [6]. It has excellent mechanical properties, such as lower biodegradability than other polyesters, and it supports extracellular matrix production and cell attachment, growth, and proliferation [7]. Its properties can also be modified for use in specific applications [8].

Potential cell sources for cartilage production using bioengineering techniques include chondrocytes, fibroblasts, and stem cells. It is necessary to increase the number of cells through subculture before implantation into the scaffold [9].

Scaffolds must support cell attachment, proliferation, and division, so it is crucial to fabricate a scaffold that has a physical structure and composition resembling an extracellular matrix [10]. Among other conditions, the pore size and pore interconnectivity of the scaffold are crucial to new tissue cell penetration, cell migration, nutrient diffusion, and the removal of metabolic substances. Ultimately, the scaffold provides a 3-dimensional (3D) microenvironment that induces cell assembly and differentiation [11]. However, the pore size and interconnectivity necessary to induce optimal cell growth differ across cell types, and research in this area is ongoing.
In this study, we conducted a morphologic evaluation of chondrocytes and fibroblasts and observed cellular interactions to determine the most suitable scaffold pore size for the production of 3D patient-specific implants.

\section{METHODS}

\section{Fabrication of 3D PCL scaffold}

The $3 \mathrm{D}$ printing was used to fabricate PCL-based scaffolds with pore sizes of 100, 200, 300, and $400 \mu \mathrm{m}$ for disc-shaped implants (diameter, $15 \mathrm{~mm}$; height, $10 \mathrm{~mm}$ ) (Fig. 1). The diameter of the nozzle and the height of the stack were both $250 \mu \mathrm{m}$. The scaffolds were manufactured in a clean-room environment that was compliant with International Organization for Standardization (ISO) 14644 standards. Per ISO 11137 guidelines, each scaffold was sterilized with gamma radiation. Scaffolds were considered acceptable for use after being subjected to strict quality control (under Osteopore's ISO 13485-compliant quality management system), the standards for which included maintenance of a porosity of $70 \% \pm 5 \%$. To maintain this porosity, scaffolds were stacked at alternating angles in a layer-by-layer laydown pattern (Fig. 2). The pore sizes of the PCL scaffolds were $100,200,300$, and $400 \mu \mathrm{m}$, and two sets of each scaffold were created.

\section{Morphologic evaluation of PCL scaffolds}

Morphologic evaluations of pore size were performed with an S-4800 scanning electron microscope (SEM; Hitachi, Tokyo, Japan) after cutting each specimen into a $5 \times 3 \times 1 \mathrm{~mm}$ cube, before seeding the scaffold with chondrocytes and fibroblasts. After fixing, the specimen was coated with platinum using an SC

\section{Fig. 1. Three-dimensional printed polycaprolactone (PCL) scaffold}

(A, B) Pore sizes of 100, 200, 300, and $400 \mu \mathrm{m}$ for disc-shaped implants.

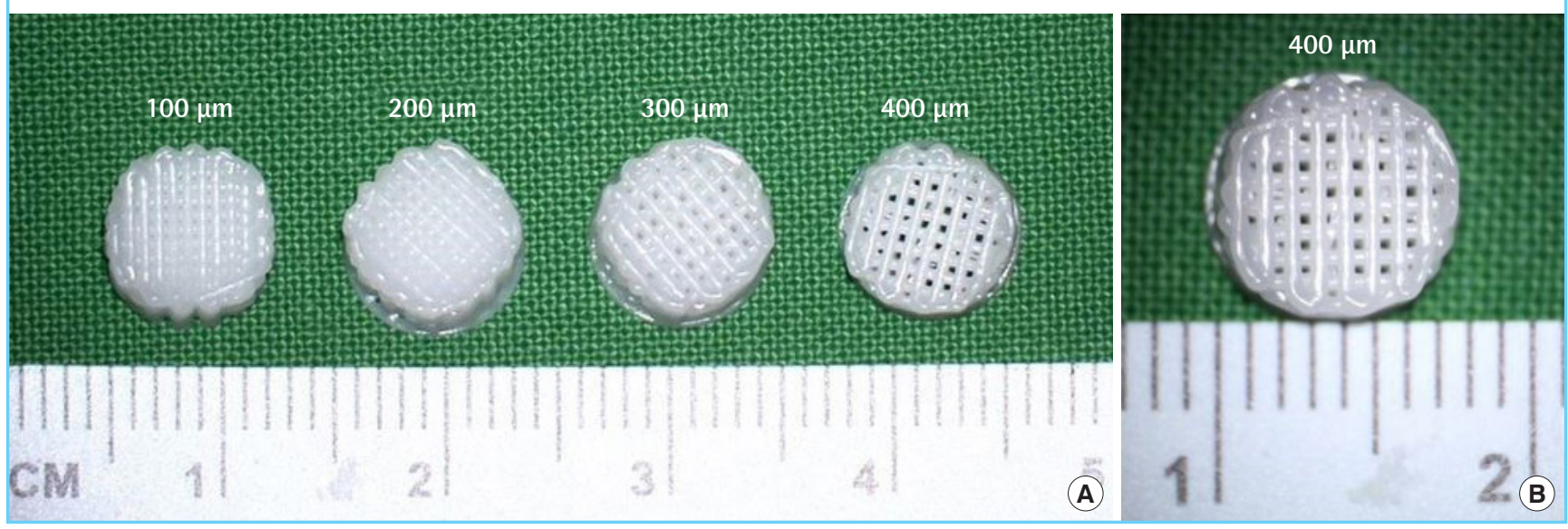




\section{Fig. 2. Method of producing a polycaprolactone (PCL) scaffold \\ A binder material was deposited onto beds with an inkjet printer head, layer by layer.}

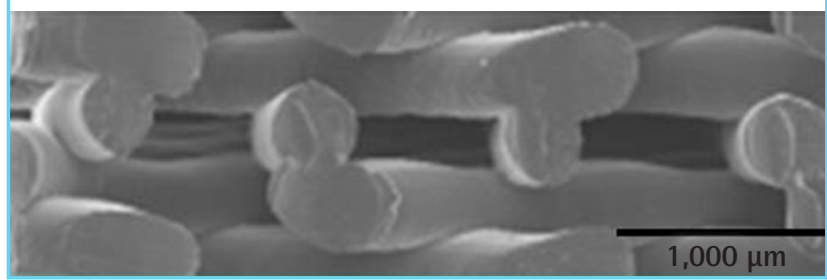

500K plasma sputter coater (Emscope, West Sussex, UK) and observed at $15 \mathrm{kV}$.

\section{Animal model}

All animal experiments were carried out in alignment with the Guidelines for the Care and Use of Laboratory Animals. Male Institute of Cancer Research mice aged 4 to 5 days (Orient Bio Inc., Seongnam, Korea) were used.

\section{Chondrocyte isolation}

A total of 12 animals were anesthetized with peritoneal anesthesia using $0.008 \mathrm{~mL}$ per $10 \mathrm{~g}$ of Zoletil (Virbac, Carros, France) and $0.002 \mathrm{~mL}$ per $10 \mathrm{~g}$ of Rompun (Bayer, Leverkusen, Germany). The costal and femoral articular cartilage was harvested through meticulous dissection and minced finely (Fig. 3). The cartilage was then washed with phosphate-buffered saline (PBS), after which it was digested with $0.2 \%(\mathrm{w} / \mathrm{v})$ collagenase (Worthington Biochemical, Lakewood, NJ, USA) in PBS for 5 hours at $37^{\circ} \mathrm{C}$. The cells were filtered using a $100-\mu \mathrm{m}$ Nylon cell strainer (Falcon; BD Biosciences, Franklin Lake, NJ, USA) and were then pooled and centrifuged at 1,700 rpm for 10 minutes. After two rounds of washing with PBS, the cell pellet was suspended in Dulbecco modified Eagle medium (DMEM; Gibco, Grand Island, NY, USA) supplemented with $10 \%$ fetal bovine serum (Gibco), 1\% penicillin G (Sigma-Aldrich, St. Louis, MO, USA), and $1 \%$ gentamicin sulfate (Sigma-Aldrich). The presence of chondrocytes was confirmed via Safranin O staining.

\section{Fibroblast isolation}

A total of four animals were sacrificed, and the anesthetic procedure was the same as above. Skin from the animals was harvested, and the muscle and adipose tissue were removed via scraping of the dermal side with a sterile scalpel. The tissue was rinsed with a $0.25 \%$ trypsin-EDTA (ethylenediaminetetraacetic acid) solution (Gibco), and an initial digestion buffer was prepared by mixing trypsin and Dispase (BD Biosciences) at a 1:1 ratio. The dermis was minced into small pieces, and a second digestion buffer consisting of $0.25 \%$ collagenase/FAD (flavin ade-

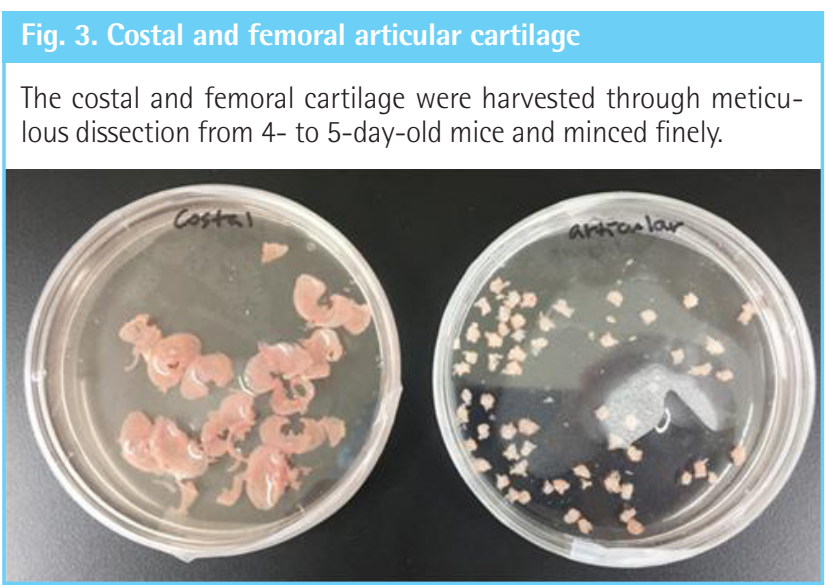

nine dinucleotide) and Ca was prepared by mixing $0.125 \mathrm{~g}$ of collagenase type I with $50 \mathrm{~mL}$ of DMEM/F12 medium (Gibco). The suspension of dermal cells was passed through a 70- $\mu \mathrm{m}$ filter using complete DMEM (Gibco), 20\% fetal bovine serum (Gibco), and 1\% penicillin-streptomycin (Sigma-Aldrich). The dish was then incubated at $37^{\circ} \mathrm{C}$ in an atmosphere of $5 \% \mathrm{CO}_{2}$ for 3 days without disturbance.

\section{Cell seeding and method of storage}

Ethylene oxide-sterilized PCL scaffolds with 100-, 200-, 300-, and $400-\mu \mathrm{m}$ pores were prepared in 48-well polystyrene dishes (Corning Inc., Corning, NY, USA). Then, the isolated chondrocytes and fibroblasts (cell density, $1 \times 10^{6}$ cells/scaffold) were seeded using pipettes in each scaffold section. To promote cell adhesion, cell-seeded scaffold sections were incubated at $37^{\circ} \mathrm{C}$ in a humidified, $5 \% \mathrm{CO}_{2}$ atmosphere. The scaffold section was transferred to a new 48-well polystyrene dish, $3.0 \mathrm{~mL}$ of culture medium was added to each well, and the plate was incubated for 8 weeks interspersed with mild shaking at approximately 50 rpm.

\section{Method of observation: MTT assay}

The viable cell counts in the scaffolds after culture for 2, 7, 14, 28, and 56 days were estimated using an MTT assay. To perform this assay, each scaffold was rinsed three times with PBS and then transferred to a 48 -well culture dish. Then, $500 \mathrm{~mL}$ of MTT stock solution (Sigma-Aldrich) was placed in each well along with $5 \mathrm{~mL}$ of culture medium and incubated at $37^{\circ} \mathrm{C}$ for 3 hours. After removing the culture medium, the formazan precipitates of the viable cells were solubilized via addition of $2 \mathrm{~mL}$ of $0.1 \% \mathrm{HCl}$ with $45 \%$ dimethyl formamide and $10 \%$ sodium dodecyl sulfate for 12 hours. A precision microplate reader (Molecular Devices, San Jose, CA, USA) was then used to determine the optical density of formazan at $450 \mathrm{~nm}$. The viable cell count was calculated using a linear calibration curve of the 
optical density plotted against preset cell concentrations. Data were collected and averaged from the scaffolds for each pore size. The cells in the scaffolds were additionally observed under SEM; to accomplish this, the attached cells were fixed with $2.5 \%$ glutaraldehyde (Sigma-Aldrich) for 30 minutes at room temperature. After careful rinsing with PBS, each of the scaffolds with its attached cells was dehydrated in a graded series of ethanol $(50 \%, 60 \%, 70 \%, 80 \%, 90 \%$, and $100 \%)$ for 10 minutes and left to dry on a clean surface at room temperature. The surfaces and cross-sections of the scaffolds were examined with a model S-4800 SEM (Hitachi) after osmium coating [12].

\section{RESULTS}

\section{Morphologic evaluation}

On day 2, the cells were evenly seeded on the surfaces of all scaffolds, and no cell growth or differences among the scaffolds were observed. The cells became attached to the surface of the scaffold and gradually proliferated and colonized the surface by day 14. Cell-to-cell interaction was not clearly observed among the chondrocytes, but it was actively observed among the fibroblasts starting on day 14 . However, there were no meaningful differences observed at the 14-day mark regardless of pore size or cell type. On day 28, cell-to-cell interaction was observed in the chondrocytes, and a greater degree of cell proliferation and interaction was observed on the scaffolds with larger pore sizes for both chondrocytes and fibroblasts. On day 56, the scaffolds (with the exception of the scaffold with the $100-\mu \mathrm{m}$ pore size) were observed to be covered with proliferated chondrocytes to the extent that their surfaces were not visible. Fibroblasts were also observed to completely cover the scaffold surface at 300 and $400 \mu \mathrm{m}$, whereas slow cell growth was observed at 100 and $200 \mu \mathrm{m}$ (Fig. 4).

\section{Quantitative evaluation: MTT assay}

The cells $\left(1 \times 10^{6}\right.$ cells/scaffold $)$ were seeded in each scaffold, and the scaffolds were checked on day 2 to ensure that the cells had been injected evenly. At that point, the number of cells was found to be relatively even, indicating a similar degree of loss across scaffolds. As time passed, it was evident that cells did not die and continued to proliferate. No noteworthy difference was observed in the number of cells for both fibroblasts and chondrocytes until day 14 . On day 28 , the chondrocyte counts showed moderate differences; however, except for the 100- $\mu \mathrm{m}$ scaffold, no remarkable differences were observed. With regard to fibroblasts, the cell counts increased in a similar fashion, but the increase was greater at 300 and $400 \mu \mathrm{m}$ than at the lower pore sizes. On day 56, it was evident that the larger the pore size, the greater the cell count. The SEM results confirmed that the morphologic evaluation aligned with these observations (Fig. 5).

\section{DISCUSSION}

As rhinoplasty has progressed and improved, various techniques and implants have been developed. Alloplastic implants are associated with problems such as foreign body reactions, and autologous implants in turn have issues of donor site morbidity and lack of source tissue. The potential of 3D implants using autologous tissue has been realized due to the development of tissue engineering technology and medical technology. These implants are of major interest in augmentation rhinoplasty.

Broadly, 3D culture methods can be categorized into scaffold

\section{Fig. 4. Scanning electron microscope images $(\times 350)$}

(A) Fibroblasts and (B) chondrocytes attached to the surface of a polycaprolactone (PCL) scaffold at different time points.
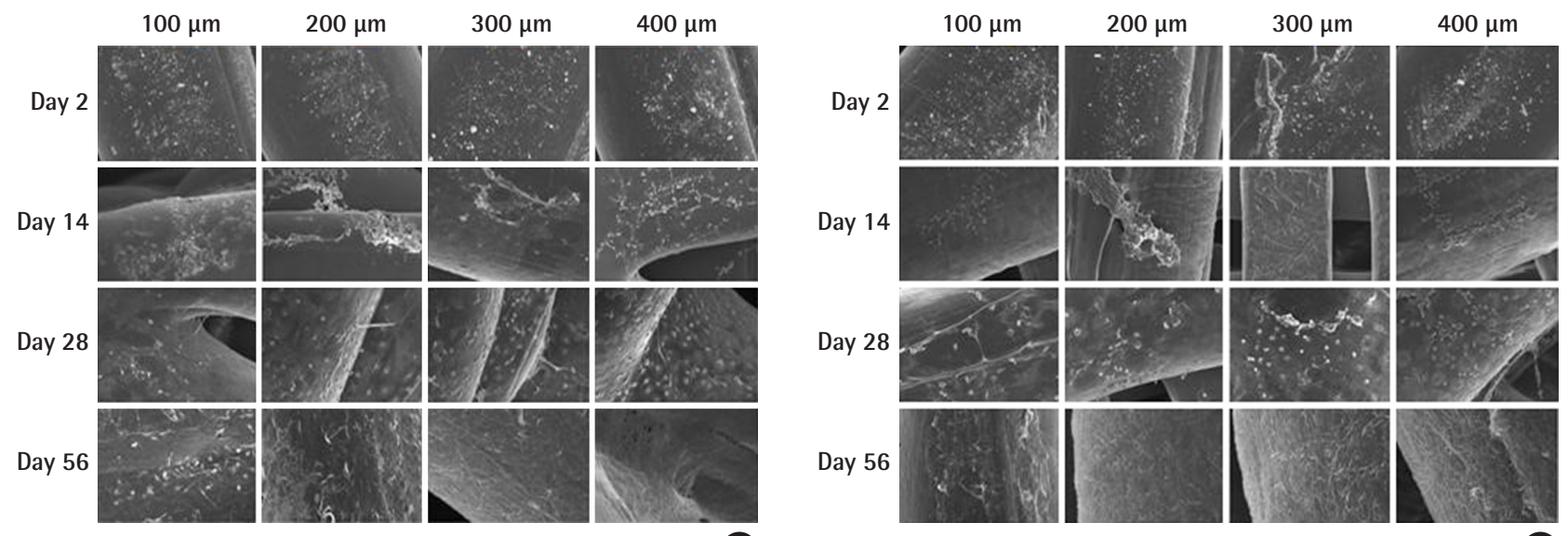


\section{Fig. 5. Cell counts for cell culture time}

Cell counts of chondrocytes (A) and fibroblasts (B).
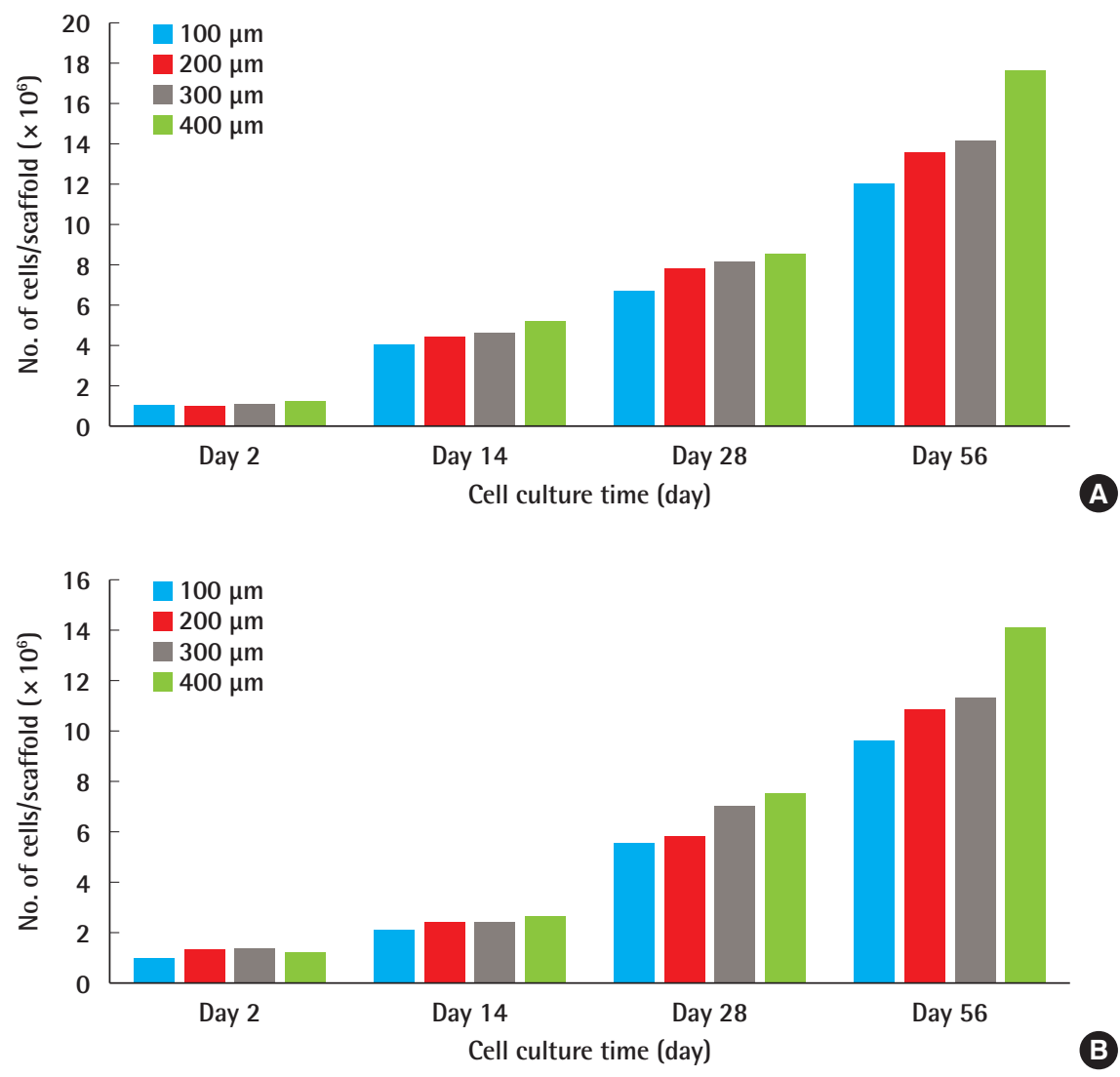

and scaffold-free techniques. The former involves the utilization of solid scaffolds, hydrogels, and other materials, while the latter makes use of methods other than the use of a scaffold, including micropatterned surfaces, low-adhesion plates, rotating bioreactors, and magnetic 3D bioprinting. Porous scaffolds form a 3D microenvironment in which cells can retain their natural structures as opposed to becoming flattened, as in conventional twodimensional cell culture. More crucially, the cells can contact and interact with adjacent cells within the scaffold to create a tissue-like structure [13].

In 3D implant production, the scaffold plays a key role in the tissue culture, and the pore size of the scaffold is crucial to cell binding, migration, growth, and regeneration and is directly related to overall function. In general, large pore sizes are effective in nourishing, diffusing and eliminating metabolites, but they are not ideal for cell binding or intracellular signaling $[12,14]$. In addition to the pore size, the materials applied to make the scaffold are important. Thus, it is important to determine the pore size at which cells can exhibit optimal growth, and developing biomedical products accordingly will improve the results in the experiments and procedures in which these scaffolds are used.

Biomaterial currently used for 3D tissue engineering can be classified into natural polymers (e.g., silk fibroin, keratin, gelatin, collagen, elastin, fibrinogen, chitosan, and alginate), synthetic polymers (e.g., polyglycolic acid, polylactic acid, and PCL), inorganic biomaterials (e.g., titanium, alumina, and zirconia), and hybrid combinations of the above (e.g., PCL with silk fibroin). These compounds must be mechanically stable with proper biodegradability and porosity, facilitating the transport of nutrients, gases, and byproducts. Natural and synthetic polymers are used for tissue engineering and regeneration due to their wide array of characteristics, such as biodegradability, high porosity, high surface-to-volume ratio, and small pore size.

Certain inorganic biomaterials have been used to replace periodontal abnormalities and musculoskeletal system damage [15]. Biomaterials composed of a hybrid of organic and inorganic components can be multifunctional and customizable with regard to their structural, thermal, and mechanical stability [16].

Previous studies have demonstrated the safety of the 3D-fabri- 
cated nasal implant $(40 \times 10 \mathrm{~mm}$; thickness, $0.8 \mathrm{~mm}$ or $1.0 \mathrm{~mm}$; porosity, $50 \%$, with $500-\mu \mathrm{m}$ pores) using PCL [17], so the authors of the present study chose to fabricate scaffolds using PCL.

Factors that can affect tissue engineering within a scaffold include the size of the pores, the porosity, the interconnectivity between pores, the shape, the material surface chemistry, the effective scaffold degradability, and the scaffold stiffness [18].

If the pores are too small, cell interaction, migration, and metabolism will be limited, promoting the death of the cells attached to the scaffolds. In contrast, if the pores are too large, the surface area available for cell attachment is reduced such that the mechanical strength of the scaffold is destroyed due to increased void volume [19]. According to Perez and Mestres [20], the ideal scaffold pore size falls between 100 and $400 \mu \mathrm{m}$. Pores larger than $500 \mu \mathrm{m}$ are too large to facilitate cell interaction with the scaffold under static seeding conditions; thus, cells penetrate the scaffolds without attaching to them. Several studies have been published regarding optimal pore size ranges for various cell and tissue types, such as $70-120 \mu \mathrm{m}$ for chondrocyte ingrowth [12], 40-150 $\mu \mathrm{m}$ for fibroblast binding [21], and 200$350 \mu \mathrm{m}$ for osteoconduction [22]. Therefore, the authors of the present study chose to test scaffolds with 100-, 200-, 300-, and 400- $\mu \mathrm{m}$ pore sizes.

The biomaterial used can also influence cell growth and attachment. Griffon et al. [23] reported that in a chitosan scaffold, chondrocytes grew best at the 70 - to $120-\mu \mathrm{m}$ pore size. In a separate study, a poly (urethane urea) scaffold with pores of 150 $300 \mu \mathrm{m}$ exhibited more chondrocyte proliferation after 28 days of in vitro culture than scaffolds with either $<150-\mu \mathrm{m}$ or 300 - to $500-\mu \mathrm{m}$ pores [24]. Given these findings, it can be concluded that the scaffold material plays a crucial role in the effects of pore size. Thus, when manufacturing $3 \mathrm{D}$ implants, it is necessary to fabricate the scaffold pore size with consideration of the material-to-cell interactions.

Oh et al. [9] published an article in 2006 discussing a cylindertype PCL scaffold with a pore size gradient. These authors set the pore size to increase from the bottom $(88 \mu \mathrm{m})$ to the top $(405 \mu \mathrm{m})$ of the scaffold. The scaffold section with a pore size of 380 to $405 \mu \mathrm{m}$ displayed superior cell growth of chondrocytes, while the section with a pore size of 186 to $200 \mu \mathrm{m}$ better facilitated fibroblast growth. The results regarding fibroblasts differed from the findings of our experiments, which can be attributed to the use of a cylinder-shaped scaffold by Oh et al. compared to the disc-shaped scaffold utilized in the present study. In other words, it seems that differences were present with regard to cell penetration into the $3 \mathrm{D}$ scaffold, and it follows that the shape of the scaffold may influence cell attachment and growth.
In addition to the research on scaffold pore size and morphology, efforts are ongoing to improve the attachment of cells to the scaffolds. Indeed, fibrin gel and collagen promote cell attachment and exhibit excellent biocompatibility [25]. Advances have also been made in tissue engineering to induce ingrowth into the scaffold in addition to surface growth.

In summary, disc-shaped scaffolds with 100-, 200-, 300-, and $400-\mu \mathrm{m}$ pores were fabricated using PCL, and chondrocytes and fibroblasts were seeded and cultured with mild shaking until 56 days had elapsed. When analyzed by an MTT assay and SEM, it was confirmed that the cells displayed the greatest amount of proliferation in the $400-\mu \mathrm{m}$ scaffold.

Through this study, we have contributed to research on the use of a 3D culture technique for implant production. We expect that through in vivo studies, the practical use of customized patient-specific implants will be achieved and will serve as a new development in plastic surgery.

\section{NOTES}

\section{Conflict of interest}

No potential conflict of interest relevant to this article was reported.

\section{Ethical approval}

All animal experiments were carried out in alignment with the Guidelines for the Care and Use of Laboratory Animals of Soonchunhyang University Bucheon Hospital (IACUC No. SCHBC-2016-11).

\section{Author contribution}

Conceptualization: JH Nam, ES Park. Data curation: JH Nam, ES Park, SY Lee, G Khan. Formal analysis: JH Nam, ES Park. Methodology: JH Nam, ES Park. Project administration: JH Nam, ES Park. Visualization: JH Nam, ES Park. Writing - original draft: JH Nam. Writing - review \& editing: JH Nam, ES Park.

\section{ORCID}

Jeoung Hyun Nam https://orcid.org/0000-0002-2151-5869 So Yun Lee https://orcid.org/0000-0002-3972-9881 Galina Khan https://orcid.org/0000-0002-2493-5279 Eun Soo Park https://orcid.org/0000-0003-2966-9122

\section{REFERENCES}

1. Ahn JM. The current trend in augmentation rhinoplasty. Facial Plast Surg 2006;22:61-9. 
2. Godin MS, Waldman SR, Johnson CM Jr. Nasal augmentation using Gore-Tex: a 10-year experience. Arch Facial Plast Surg 1999; 1:118-21.

3. Kim YS, Shin YS, Park DY, et al. The application of three-dimensional printing in animal model of augmentation rhinoplasty. Ann Biomed Eng 2015;43:2153-62.

4. Xu Y, Fan F, Kang N, et al. Tissue engineering of human nasal alar cartilage precisely by using three-dimensional printing. Plast Reconstr Surg 2015;135:451-8.

5. Yi HG, Choi YJ, Jung JW, et al. Three-dimensional printing of a patient-specific engineered nasal cartilage for augmentative rhinoplasty. J Tissue Eng 2019 Jan 16 [Epub]. https:// doi.org/10.1177/2041731418824797.

6. Baker SC, Rohman G, Southgate J, et al. The relationship between the mechanical properties and cell behaviour on PLGA and PCL scaffolds for bladder tissue engineering. Biomaterials 2009;30:1321-8.

7. Li WJ, Danielson KG, Alexander PG, et al. Biological response of chondrocytes cultured in three-dimensional nanofibrous poly( $\epsilon$-caprolactone) scaffolds. J Biomed Mater Res Part 2003;67A:1105-14.

8. Pina S, Ferreira JMF. Bioresorbable plates and screws for clinical applications: a review. J Healthc Eng 2012;3:243-60.

9. Oh SH, Park IK, Kim JM, et al. In vitro and in vivo characteristics of PCL scaffolds with pore size gradient fabricated by a centrifugation method. Biomaterials 2007;28:1664-71.

10. Park BK. Biodegradable polymers for tissue engineering: review article. J Biomed Eng Res 2015;36:251-63.

11. Nava MM, Draghi L, Giordano C, et al. The effect of scaffold pore size in cartilage tissue engineering. J Appl Biomater Funct Mater 2016;14:e223-9.

12. Oh SH, Kang SG, Kim ES, et al. Fabrication and characterization of hydrophilic poly(lactic-co-glycolic acid)/ poly(vinyl alcohol) blend cell scaffolds by melt-molding particulate-leaching method. Biomaterials 2003;24:401121.

13. Knight E, Przyborski S. Advances in 3D cell culture technologies enabling tissue-like structures to be created in vitro. J Anat 2015;227:746-56.
14. Loh QL, Choong C. Three-dimensional scaffolds for tissue engineering applications: role of porosity and pore size. Tissue Eng Part B Rev 2013;19:485-502.

15. Pina S, Ribeiro VP, Marques CF, et al. Scaffolding strategies for tissue engineering and regenerative medicine applications. Materials (Basel) 2019;12:1824.

16. Wang X, Chang J, Wu C. Bioactive inorganic/organic nanocomposites for wound healing. Appl Mater Today 2018;11: 308-19.

17. Park YJ, Cha JH, Bang SI, et al. Clinical application of threedimensionally printed biomaterial polycaprolactone (PCL) in augmentation rhinoplasty. Aesthetic Plast Surg 2019;43: 437-46.

18. Yamane S, Iwasaki N, Kasahara Y, et al. Effect of pore size on in vitro cartilage formation using chitosan-based hyaluronic acid hybrid polymer fibers. J Biomed Mater Res A 2007;81: 586-93.

19. Im GI, Ko JY, Lee JH. Chondrogenesis of adipose stem cells in a porous polymer scaffold: influence of the pore size. Cell Transplant 2012;21:2397-405.

20. Perez RA, Mestres G. Role of pore size and morphology in musculo-skeletal tissue regeneration. Mater Sci Eng C Mater Biol Appl 2016;61:922-39.

21. Salem AK, Stevens R, Pearson RG, et al. Interactions of 3T3 fibroblasts and endothelial cells with defined pore features. J Biomed Mater Res 2002;61:212-7.

22. Whang K, Healy KE, Elenz DR, et al. Engineering bone regeneration with bioabsorbable scaffolds with novel microarchitecture. Tissue Eng 1999;5:35-51.

23. Griffon DJ, Sedighi MR, Schaeffer DV, et al. Chitosan scaffolds: interconnective pore size and cartilage engineering. Acta Biomater 2006 May;2:313-20.

24. Stenhamre H, Nannmark U, Lindahl A, et al. Influence of pore size on the redifferentiation potential of human articular chondrocytes in poly(urethane urea) scaffolds. J Tissue Eng Regen Med 2011;5:578-88.

25. Li Y, Meng H, Liu Y, et al. Fibrin gel as an injectable biodegradable scaffold and cell carrier for tissue engineering. ScientificWorldJournal 2015;2015:685690. 